\title{
How infant-directed actions enhance infants' attention, learning, and exploration: Evidence from EEG and computational modeling
}

\author{
Marlene Meyer ${ }^{1,2^{*}}$, Johanna E. van Schaik ${ }^{3}$, Francesco Poli ${ }^{1} \&$ Sabine Hunnius ${ }^{1}$ \\ ${ }^{1}$ Donders Institute for Brain, Cognition and Behaviour, Radboud University Nijmegen, The Netherlands \\ ${ }^{2}$ Department of Psychology, University of Chicago, USA \\ ${ }^{3}$ Behavioural Science Institute, Radboud University Nijmegen, the Netherlands
}

\begin{abstract}
Author Contributions
MM, JEvS and SH jointly developed the study concept and design. MM and JEvS collected the data. MM and JEVS performed EEG data analyses, and MM and FP performed computational modelling analyses.

All authors interpreted the data. MM drafted the manuscript and all authors contributed critical revisions. All authors approved the final version of the manuscript for submission.
\end{abstract}

\section{${ }^{*}$ Corresponding Author}

marlene.meyer@donders.ru.nl

Thomas van Aquinostraat 4, 6525 GD, Nijmegen

The Netherlands 


\section{Keywords}

EEG, Theta Oscillations, Variability, Infants, Attention, Infant-directed Actions, Surprise, Computational modeling

\section{Highlights}

- 15-month-olds watched actions with normal, high, and variable movement amplitude

- Infants show increased frontal theta power when actions contain variable movements

- Frontal theta during variable movements relates to infants' subsequent learning and exploration

- Computational modelling suggests that surprise, induced by variability, drives theta 


\section{Abstract}

When teaching infants new actions, parents tend to modify their movements. Infants prefer these infantdirected actions (IDAs) over adult-directed actions and learn well from them. Yet, it remains unclear how parents' action modulations capture infants' attention. Typically, making movements larger than usual is thought to draw attention. Recent findings, however, suggest that parents might exploit movement variability to highlight actions. We hypothesized that variability in movement amplitude rather than higher amplitude is capturing infants' attention during IDAs. Using EEG, we measured 15-month-olds' brain activity while they were observing action demonstrations with normal, high, or variable amplitude movements. Infants' theta power $(4-5 \mathrm{~Hz})$ in fronto-central channels was compared between conditions. Frontal theta was significantly higher, indicating stronger attentional engagement, in the variable compared to the other conditions. Computational modelling showed that infants' frontal theta power was predicted best by how surprising each movement was. Thus, surprise induced by variability in movements rather than large movements alone engages infants' attention during IDAs. Infants with higher theta power for variable movements were more likely to perform actions successfully and to explore objects novel in the context of the given goal. This highlights the brain mechanisms by which IDAs enhance infants' attention, learning, and exploration. 


\section{Introduction}

When showing novel actions to their infants, such as how to use a new rattle or how to stack blocks, parents tend to modify their movements. Infant-directed actions (IDAs) differ from adult-directed actions (ADAs) in movement amplitude and speed, in the number of repetitions (Brand, Baldwin \& Ashburn, 2002; van Schaik et al., 2020), in the number of toy exchanges, and in the duration of action effect demonstrations (Brand, Baldwin \& Ashburn, 2002; Brand, Shallcross, Sabatos, \& Massie, 2007; van Schaik et al., 2020). What is the function of this phenomenon that seems counterintuitive at first, as parents are demonstrating a version of the action not identical to the one to be learned by the infant? The key to understanding this phenomenon likely is the functional role IDAs have for infants' attention and learning. Like infant-directed speech (ManyBabies Consortium, 2020), IDAs enhance infants' attention to the demonstrated action (Brand \& Shallcross, 2008; Koterba \& Iverson, 2009) and facilitate action learning (Schreiner, van Schaik et al., 2020; Williamson \& Brand, 2014). Evidence for such attentional enhancement comes from looking time studies with infants and young children. Brand and Shallcross (2008) tested 6to 8-month-olds and 11- to 13-month-olds in a preferential looking paradigm, presenting them with IDAs and ADAs. Their results show that infants of both age groups preferred looking at IDAs compared to ADAs. Similarly, Koterba and Iverson (2009) investigated the looking behavior of 8- to 10-month-old infants when observing their caregiver demonstrate actions on novel toys. In this study, parents were instructed to demonstrate an action with high or low amplitude movements and repeat it or not. Infants looked longer at IDAs if they were performed with high amplitude movements, were repeated or had high amplitude movements which were repeated, compared to non-repeated low amplitude movements.

In addition to enhanced attention during IDAs, several studies have demonstrated that IDAs affect infants' action exploration and learning (Koterba \& Iverson, 2009; van Schaik et al., 2020; Williamson \& Brand, 2014). For instance, Williamson and Brand (2014) assessed the imitation performance of 2-year- 
Surprise induced by variability in IDA enhances infants' attention

old children; a baseline group who did not see any action demonstrations, an ADA group and an IDA group. Children who had IDA demonstrations were more likely to perform the demonstrated action compared to the ADA and baseline groups. While this body of research suggests that IDAs play an influential role in infants' attention to and learning of actions, it is unknown how IDAs capture infants' attention and guide their learning.

\section{How do IDAs draw infants' attention?}

One prototypical feature associated with IDAs is the use of larger movements than usual. One possibility is, therefore, that IDAs draw infants' attention because they are larger than normal actions. Studies in which adults were instructed to make large movements when demonstrating an action found attentional and learning effects in infants (Koterba \& Iverson, 2009; Williamson \& Brand, 2014). However, movement amplitude was manipulated together with other features, like repetition, emotional engagement, and proximity to the infant (Koterba \& Iverson, 2009; Williamson \& Brand, 2014). Two other findings render it less likely that large movements are the driving force of infants' attention during IDAs. That is, Koterba and Iverson (2009) saw increased looking time to IDAs performed repeatedly with small amplitude compared to a control condition, in another study, and large movements were found to be dependent on the action at hand rather than universal across actions (van Schaik et al., 2020). While making large movements remains a possible factor underlying infants' attention to IDAs, recent findings suggest another potential driving factor: variability in movement.

In a study by Fukuyama and colleagues (2015) parents were found to dynamically modulate the variability in their IDAs dependent on their children's behavior. More specifically, the authors measured mothers' movement kinematics and their 11- to 13-month-olds' behavioral performance during a dyadic interaction in which mothers demonstrated a cup-nesting action. Mothers increased the variance in their movements after their infant had performed irrelevant actions with the cups in between demonstrations 
and decreased their movement variance after their infant performed the target actions. This suggests that movement variability might be exploited by parents to direct their infants' attention to the demonstrated action when needed. Performing movements that deviate from the usually small and efficiently performed movements parents make might represent such a natural variation as well. Thus, movement variability and amplitude remain confounded in everyday life. Furthermore, this role of variability is supported by findings from other lines of research, such as statistical learning work which suggests that infants pay more attention to variable, less predictable input (Johnson \& Munakata, 2005; Tummeltshammer \& Kirkham, 2013). In sum, instead of solely large movement amplitudes, variability in movements could be responsible for capturing infants' attention, thereby influencing learning and exploration in IDAs.

\section{The current study}

In this EEG study, we investigated the roles of variability and movement amplitude in enhancing infants' attention to IDAs. We hypothesized that variability in movement attracts infants' attention more than large movements per se. We also investigated the relation between infants' attention and their learning.

So far, studies on IDAs have relied on looking time measures as proxy for infants' attention. In the current study, we made use of a neural indicator of infants' attention which allows for a more sensitive measure which does not dependent on behavioral changes. More specifically, we examined modulations in frontal theta band oscillations. Theta band activity in frontal brain regions has previously been linked to top-down and bottom-up attention in infants (Begus, Southgate \& Gliga, 2015; Orekhova et al., 2006), young children (Meyer et al., 2019), and adults (Clayton, Yeung, \& Kadosh, 2015). Frontal theta power has been proposed to signal the need for cognitive control (Cavanagh \& Frank, 2014), often elicited by attention capturing events. Frontal theta band modulations are also associated with memory processes (Jensen \& Tesche, 2002; Begus Southgate \& Gliga, 2015) and are thought to reflect infants' learning of new 
Surprise induced by variability in IDA enhances infants' attention

information (Begus \& Bonawitz, 2020). For instance, findings on neural processing of infant-directed speech provide evidence for an increase in infants' frontal theta power when listening to infant-directed speech compared to control conditions (Orekhova et al., 2006; Zhang et al., 2011). Thus, frontal theta band power is a promising neural measure to assess infants attentional processing during IDAs.

Our experimental set-up consisted of a demonstration and an exploration phase. We measured 15-month-old infants' brain activity while they observed action demonstrations executed with normal, high, and variable movement amplitudes. After the demonstration phase, infants had the opportunity to perform the actions themselves. To investigate how the different conditions affected infants' attentional processing, we compared infants' theta power $(4-5 \mathrm{~Hz})$ in fronto-central midline channels $(\mathrm{Fz}, \mathrm{FCz}, \mathrm{Cz})$ between conditions, controlling for multiple comparisons. In addition, we computed a linear regression model to examine the link between infants' attention as reflected by their frontal theta power to variable amplitude movements and their exploration and learning behavior. As an exploratory analysis, we further investigated which aspect of the movement variability might be driving any potential effects. Variable movements are both, more surprising and more complex. To disentangle whether surprise or complexity better described infants' frontal theta power, we additionally used a computational modelling approach. 


\section{Material and Methods}

\section{Participants}

In the final sample 23 infants (10 girls) are included. Infants' mean age was 15.9 months (range $=15.5$ to 16.5 months). We chose this age on the basis of previous research on IDAs showing that caregivers modulate their movements towards infants of this age and showing that around this age infants are capable of imitating basic actions, an important aspect for the learning part of this study (van Schaik et al., 2020). Another 24 infants were tested of which 12 had to be excluded due to an error in the automatized randomization, 5 were excluded because of insufficient EEG recording quality and another 7 participants did not contribute sufficient artifact-free trials to the analysis (see EEG analysis for details). Families who participated in the study came from a middle-sized Dutch city and were recruited from a database of families volunteering in developmental research. The current study adheres to the Declaration of Helsinki and was approved by the local ethics board. Parents gave written consent to their infant's participation in the study. As compensation for their travels and time, families could choose to receive either two children's books, a children's book and 10 euros, or 20 euros.

\section{Stimuli}

For this study, we created avatar stimuli based on motion-tracking recordings of an adult model (see Figure 1). The advantage of using avatars and a virtual environment instead of video recordings was the precise manipulation of movement amplitude in the stimuli. That is, it allowed us to create instances of low and high amplitude movements with human kinematic features that could be combined into different stimulus movies. Using a Qualisys motion-tracking system (a Qualisys Oqus 5 + system with seven infrared cameras for motion-tracking and one video camera) we recorded the movements of an adult model performing three different actions; stacking rings on a peg, building a tower with cups and putting balls in 
a bucket. For each of these actions, the model reached out to, picked up, and moved the target object (e.g., ring) to the corresponding goal base (e.g., peg). The model performed the same action five times, for instance putting five rings on the peg. Crucially, once converted to avatar stimuli, we manipulated the amplitude of the goal-directed movements which preceded the final step of each action (e.g., the amplitude of lifting the ring to put it on the peg), resulting in two versions of each action, one with a normal amplitude and one with a high amplitude.

From this manipulation, we made stimulus videos in three different conditions (see Figure 2) that is five normal amplitude movements, five high amplitude movements, and five variable amplitude movements for each action type (using balls, rings, cups). We created the variable condition by combining the high and normal amplitude movements into different orders (e.g., a sequence of normal, high, normal, high, high; 4 pseudo-randomized sequences, counterbalanced across participants).

Each stimulus video with five movements lasted for about 32 seconds including an initial 2 seconds still frame phase followed by the five goal-directed movements of one action type. The avatar was shown with a cap that covered the upper half of the face to ensure infants would pay attention to the action rather than the actor's face and gaze. The initial visual scene of each experimental video included the model making eye-contact and sitting at a table with all five target and ten distractor objects as well as the specific goal base in front of her (see Figure 1). The objects and bases used in the stimuli were also available for infants to explore in real life at the test session (see Procedure for details).

Additionally, we created two other types of video stimuli, intro videos and peekaboo videos. A short intro videos was made in which the infant was greeted by the actor saying "Hey, baby". The intro video lasted for about 2 seconds and preceded each of the experimental videos. Besides this, peekaboo videos were recorded with the actor playing the classic peekaboo game by hiding her face behind her hands for 1-4 seconds. The peekaboo videos served as attention getters between trials. Together the 
intro, the experimental and the peekaboo videos made up blocks of trials as described in more detail in the Procedure section.

\section{Procedure}

Parent and infant were invited to the lab for a combined EEG and behavioral testing session. At the beginning of the session, parents were informed about the procedure by one experimenter (E1) while another experimenter (E2) played with the infant. This introduction also allowed the infant to become familiarized with the new environment. After parents gave written consent for their infant's participation in this study, one of the experimenters fitted the infant with a 32 active electrode EEG cap (actiCap, Brain Products $\mathrm{GmbH}$, Germany) while the other experimenter distracted the child with bubbles. The EEG cap was arranged in the standard 10-20 system with the online reference electrode at the left mastoid. We strived to get impedances of all electrodes below $60 \mathrm{k} \Omega$. To ensure that all electrodes touched the infant's head and to prevent infants from reaching and pulling the electrode cables the experimenter additionally placed a tubular elastic bandage (Surgilast) over the EEG cap and electrodes.

Once the EEG cap was prepared the experimenters accompanied parent and infant into an electronically shielded EEG testing booth. Parents were then seated in front of a screen (about $60 \mathrm{~cm}$ distance) with their infant on their lap (see Figure 3). When parent and infant were seated comfortably, the experimenters left the room and the experimental testing session started. Infants' EEG was amplified using a BrainAmp DC EEG amplifier, digitized at $500 \mathrm{~Hz}$ and recorded with a band-pass filter of .1 to $200 \mathrm{~Hz}$ using BrainVision Recorder software (Brain Products GmbH, Gilching, Germany). Additionally, the entire testing session was video recorded for later offline coding of the infant's behavior. The experiment consisted of two testing phases, the demonstration phase and the exploration phase as illustrated in Figure 3. 
During the demonstration phase, infants were presented with three actions, each shown in one of the three conditions (normal, high, and variable). Across subjects we counterbalanced which action type (acting on balls, cups, rings) was demonstrated in which condition. Each experimental video (including five goal-directed movements) were presented in 4 blocks making up a total of 20 goal-directed movements per condition. Each experimental video was preceded by a 1 second fixation cross (white cross on a grey screen) and the intro video to orient the infant to the screen. Each block contained all three experimental videos in pseudo-randomized order (i.e., one normal, one high, and one variable), followed by a peekaboo video. After the last block, all four peekaboo videos were played (with 1 to 4 seconds of hiding the actor's face) before commencing the exploration phase. All stimuli were presented using Presentation Software (Neurobehavioral Systems, Albany, CA) and the timing of the stimuli was automatically time-locked with the EEG signal.

After the demonstration phase, the EEG recording was stopped, and both experimenters entered the test booth again to present infants with the objects previously shown. In this exploration phase, infants had the opportunity to act on the objects themselves. The exploration phase had two parts, the All Objects part and the Target Objects Only part. First, each goal base was presented with target and distractor objects (All Objects part) and subsequently only with three corresponding objects (Target Objects Only part). See Figure 3 for an example. To start each exploration trial, E1 first mounted the target base in the middle of the table in front of the child. Then E1 and E2 simultaneously placed all respective objects, left and right from the base, such that the infant had access to all respective objects at the same time. Each exploration trial ended after 1 minute. After the testing session ended, the experimenters took the EEG cap off the infant and parents were informed about the purpose of the study. 
Surprise induced by variability in IDA enhances infants' attention

\section{EEG data analysis}

The EEG data were time-locked to the moment the goal of the action was reached and segmented into trials of 1 second prior to and 500ms following this time. The timing of the action goal was defined as follows in the stimuli: the first frame in which the ball hit the bottom of the bucket or the other balls, the cup was released and touched the base or the other cups, and the ring was released and touched the base or the other rings. This $1500 \mathrm{~ms}$ time window was chosen to include the movement following the peak amplitude, leading up to and finishing off the action goal without overlapping with the previous or next actions. As such the window allowed us to investigate how (changes in) movement amplitude might affect neural processing of the goal-directed action. This time window also captures 6 cycles of the lowest frequency of interest in our analysis (i.e. $4 \mathrm{~Hz}$ ), allowing for robust estimates of power in this frequency. For data padding, the data were initially epoched with an additional 500ms before and after the time window of interest.

During pre-processing, the data were first band-pass filtered between $1-30 \mathrm{~Hz}$ and the mean signal was subtracted from each time point to account for potential differences in offset. In the variable condition, the first trial did not offer any information about the variability in movement amplitude, and it was thus excluded. Based on video coding of infants' gaze behavior, trials during which infants did not look at the screen were also discarded from further analysis. Then, four rounds of artifact rejection were conducted (blind to condition) and subsequently any missing channels were interpolated. There were four rounds of artifact rejection. First, epochs with large EEG artifacts were removed based on visual rejection. Second, independent-component analysis was applied to remove components containing eye-movement artifacts. Then, two rounds of visual artifact rejection were performed to reject any remaining trials or channels with EEG artifacts. Seven participants did not provide at least 8 trials per condition and were excluded from the analyses. In the final dataset of 23 participants, an average of 15.4 trials (range: 11-20) 
in the normal amplitude condition, 15.6 trials (range: 11-20) in the high amplitude condition and 13.5 trials (range: 9-16) in the variable amplitude condition remained in the analysis.

Cleaned EEG data were re-referenced to the average mastoids and linear drift was removed from the data. A fast Fourier transform using the multitaper method (hanning taper) was computed to estimate power for each condition between 3 to $30 \mathrm{~Hz}$. Power in the theta frequency band $(4-5 \mathrm{~Hz})$ was then extracted. This frequency range is consistent with a band around the peak frequency $(4.4 \mathrm{~Hz})$ observed in previous research on infant-directed speech (Orekhova and colleagues, 2006) with infants of similar age. For comparison of theta power across conditions we ran repeated-measures ANOVAs with the factor Condition (Normal, High, Variable) for a priori defined electrodes $\mathrm{Fz}, \mathrm{FCz}$ and $\mathrm{Cz}$, applying Bonferroni correction for conducting three tests. The EEG data processing scripts are available on GitHub (https://github.com/marlenemeyer/EEG_pipeline_variability_in_infant_directed_actions).

\section{Behavioral data analysis}

The exploration phase of the experiment was video coded offline using ELAN (ELAN, 2018). In the All Objects part, we coded whether the infant first touched a distractor or a target object. The number of times the target action was successfully performed was tallied and we scored whether or not infants had performed the target action successfully at least once. For the Target Objects Only part, we also coded whether the infant performed the target action at least once, and if so, how often it was performed successfully.

\section{Analysis of relation between EEG and behavioral data}

To investigate whether there is a link between infants' attentional processing of variable movements in IDAs and their subsequent exploration and learning, we used a linear regression model. In the model, we examined the relation between frontal theta power during the variable action demonstration and the 
following, simultaneously entered variables: Object First Touched (i.e. the object that was first touched, target or distractor) from the All Objects part, together with Successful Target Performance (i.e. whether or not the target action was performed at least once) from both exploration parts separately. All behavioral measures were taken during the time infants could explore the objects together with the goal base matching the variable condition. Due to both the focus of this study being on the variable condition and the limited data spread of frontal theta power at Fz in the high and normal conditions, regression models for these conditions were not included in the main manuscript. However, for completeness, they are available in the Supplementary Material (Tables S1 and S2). 


\section{Results}

\section{EEG results}

Figure 4 illustrates the grand average power across participants at pre-defined channels $\mathrm{Fz}, \mathrm{FCz}$, and $\mathrm{Cz}$ as a function of frequency $(3-30 \mathrm{~Hz}$ ) and condition (normal, high, variable). In addition to the inherent $1 / \mathrm{f}$ distribution of power, the figure shows a frequency-specific modulation of condition in the theta band (4$5 \mathrm{~Hz}$ ). That is, power in the theta band is higher for the variable compared to both the normal and high amplitude movement conditions. This difference appears to decrease from frontal to central sites. Figure 5 shows boxplots of the extracted theta power values per condition for each of the channels. A repeatedmeasures ANOVA comparing theta power $(4-5 \mathrm{~Hz})$ between conditions yielded a significant difference at channel $\mathrm{Fz}\left(F(2,44)=5.00, p=.011\right.$, partial eta $\left.{ }^{2}=.18\right)$. At this frontal site, theta power was highest for the variable condition $(M=10.35, S D=5.08)$ and differed significantly from the normal $(M=8.53, S D=4.31$; $t(22)=-2.318, p=.03)$ and high amplitude conditions $(M=7.84, S D=2.54 ; t(22)=-2.687, p=.01)$. No evidence for a difference in theta power was found between the normal and high conditions $(t(22)=.951$, $p=.35)$. At the fronto-central site $\mathrm{FCz}$, theta power was also descriptively higher for the variable condition $(M=10.90, S D=6.00)$ than the normal $(M=9.17, S D=4.52)$ and high conditions $(M=8.82, S D=3.32)$ but this difference did not reach significance $\left(F(2,44)=2.45, p=.09\right.$, partial eta $\left.{ }^{2}=.10\right)$. At central site $C z$, no significant difference was detected $\left(F(2,44)=1.15, p=.32\right.$, partial eta $\left.{ }^{2}=.05\right)$ between the variable $(M=$ $10.96, S D=7.61)$, normal $(M=9.56, S D=4.84)$ and high $(M=6.62, S D=4.69)$ conditions. Note that we used a Bonferroni-corrected alpha level of .016 to correct for multiple comparisons.

Figure 6 shows the topographic distribution of the theta power difference across the scalp between the variable and normal as well as the variable and high conditions. In line with indications from the three pre-defined channels, the plot suggests a frontal distribution of the effect. Together, the results 
provide evidence for an effect in the theta frequency range between conditions such that IDAs with variable movements elicit more frontal theta power than IDAs with normal or high amplitude movements.

\section{Results on the relation between EEG and behavioral data}

We used a linear regression model to examine the relation between neural measures of attention during the variable condition (theta power) and behavioral measures of exploration (Object First Touched) and, for the All Objects and Target Objects Only part, learning (Successful Target Performance). The results are represented in Table 1. For more information on the behavioral results see Supplementary Table S3. The overall linear regression model was significant $\left(F(3,21)=3.35, p=.04, R^{2}=.36\right)$. The results suggest a relation between higher theta power and successfully performing the target actions in the Target Objects Only part at least once as well as touching those objects first that were novel in the context of the given goal in the All Objects part. 


\section{Interim Discussion I}

We found that frontal theta was significantly higher, indicating stronger attentional engagement, in the variable compared to both the normal and high amplitude conditions at electrode Fz. This shows that variability in movement amplitude rather than large movements alone engages infants' attention during infant-directed actions. Whereas movements with high amplitude are considered prototypical of IDAs (e.g., Koterba \& Iverson, 2009; Williamson \& Brand, 2014), this evidence supports the idea that it is the variability of movements that parents exploit to draw their infants' attention to an action. This is consistent with previous findings in IDAs showing that mothers increased the variability in their movements if their infant was unsuccessful in performing the demonstrated action (Fukuyama et al., 2015).

We further examined whether theta power in the variable condition was related to infants' learning (indexed by correctly performing the target action) and exploration (indexed by novel object-goal associations). The results show that infants with higher theta power were more likely to successfully perform the target actions and to explore objects that were novel in the context of the given goal. One might have expected that infants with higher theta power first touch the objects corresponding to the goal base rather than objects novel in the context of that goal. Still, the findings fit well with the learning progress framework (Oudeyer, Gottlieb, \& Lopes 2016), which posits that infants engage in an activity as long as they can still learn from it, but switch to new activities when they cannot learn from the activity anymore (see also Poli et al., 2020). For infants showing higher theta power the input might have fulfilled their learning, which in turn led infants to invest their cognitive resources on exploring novel object-goal associations. Importantly, all toys (balls, cups, rings) were presented equally often and whether objectgoal associations were shown in variable, high or normal movement demonstrations was counterbalanced across participants. Therefore, this effect cannot be attributed to differences in object or goal saliency per 
se. In sum, the current exploration effect linking frontal theta power and infants' first object exploration might reflect habituation processes during the demonstration phase.

\section{What is so special about variability?}

Together, the current findings show that movement variability draws infants' attention and emphasize the role of variability for infants' attention and learning. That, however, raises the question, what is so special about variability in movement amplitude? To better understand how IDAs capture infants' attention, we examined which information entailed in variability is associated with the observed neural effect.

The variable movement condition differs from the normal and high amplitude movement conditions in multiple ways. First, variable demonstrations are more complex, containing less redundant information than IDAs with constant amplitude movement demonstrations. In a looking time study, Addyman and Mareschal (2013) investigated whether the complexity of visually presented stimulus sequences predicted infants' looking behavior. Indeed, they found that infants looked longer at sequences that were more complex as indicated by reduced local redundancy. Analogously, this might suggest that it is the stimulus complexity of the variable action demonstrations which is capturing infants' attention.

Besides being more complex, variable demonstrations are also less predictable, thus more surprising than the constant amplitude demonstrations (normal and high). The role of surprise in dyadic infant-parent interactions has also been highlighted by research on infant-directed speech. A computational modelling analysis of variability in infant-directed speech has shown that when talking to their infants, caregivers use more surprising, less predictable intonation in their speech than when talking to another adult (Räsänen, Kakouros \& Soderstrom, 2018). Given these previous findings, one might expect surprise in the variable IDAs to elicit enhanced attention in infants as indicated by frontal theta increase. 
Surprise induced by variability in IDA enhances infants' attention

From an information-theoretic perspective, both the complexity as well as the surprise level are higher in the variable condition than in the other two conditions. Therefore, whether infants are attracted by the complexity or surprise of these stimuli is an open question. To disentangle these two alternatives, we used a computational modelling approach in a post-hoc analysis to examine whether complexity or surprise in the current stimuli were predictive of infants' attentional processing as reflected in frontal theta power. 


\section{Computational Modelling Investigating the Role of Complexity}

\section{and Surprise for Infants' Attention to IDAs}

We computed the amount of complexity and surprise of each stimulus infants saw, excluding all trials on which infants looked away. As a proxy for complexity, we adopted the measure of local redundancy (Jamieson \& Mewhort, 2005) as modified and used by Addyman and Mareschal (2013):

$$
R(N)=\log \left(k_{\text {normal }} !\right)+\log \left(k_{\text {high }} !\right)
$$

where $k_{\text {normal }}$ and $k_{\text {high }}$ indicate how many times normal amplitude and high amplitude trials have occurred, and $\mathrm{N}$ indicates the maximum number of trials to be considered at each timepoint (i.e., the moving-window size). Following Addyman and Mareschal (2013), we set $\mathrm{N}=6$. An example of how local redundancy scores change over trials is illustrated in Figure 7. High values of Local Redundancy reflect low complexity in the stimulus. Local Redundancy is higher for normal and high amplitude conditions compared to the variable amplitude condition, thus capturing the difference in complexity between conditions.

As second measure of interest, we computed surprise at each trial as the amount of Shannon Information, I:

$$
I=\log _{2} p(x \mid X, \alpha)
$$

where $\mathrm{x}$ is the type of movement that occurred in the current trial (high or low amplitude), $\mathrm{X}$ consists of all the past actions that have been observed, and $\alpha$ captures the prior expectations at the start of the task. Figure 7 illustrates an example of how Surprise values change across trials for the normal, high, and variable amplitude conditions. While Surprise scores remain rather high and fluctuate on this elevated level in the variable condition, Surprise is approaching zero as trial number increases in the conditions 
Surprise induced by variability in IDA enhances infants' attention

with only normal or high amplitude movements. This indicates that variable IDAs remain surprising while repetitive normal and high amplitude IDAs quickly become predictable to the observer.

Our modeling work allows us to disentangle which of these factors is related to the variation in infants' theta power. Moreover, it is important to stress that redundancy and surprise measures make very different assumptions on how infants process IDAs. Redundancy is based on stimulus frequency, and thus it assumes that infants keep track of how often a stimulus is presented. Instead, surprise is based on probabilities, which implies that infants make probabilistic models of the world and are surprised when such models are violated.

To test whether we replicate previous research showing condition-independent increase of theta power over time (Braithwaite et al., 2020), in our analysis we also used the trial number as proxy for time. Importantly, Trial Number did not vary as a function of condition. Then, trial-based theta power at channel Fz was extracted per participant for each trial and standardized using z-transformation. Using a Cullen and Frey graph (1999) to examine our data distribution showed that a lognormal distribution fit the normalized theta power values best. Accordingly, we used generalized linear models (GLMs) to fit our data. The GLMs were fitted using the gamlss package (Stasinopoulos \& Rigby, 2007) in R. We ran models using ztransformed values of Local Redundancy, Surprise and Trial Number to estimate their relation to normalized frontal theta power. The results are represented in Table 2. The model which included Surprise and Trial Number fit the data best $(\mathrm{AIC}=2380)$ and showed a positive relation of frontal theta power with Surprise and positive relation of frontal theta power with Trial Number. This suggests that the higher the levels of surprise in the stimuli were the higher infants' theta power. Also, the more movements infants saw, reflected in higher Trial Number, the higher their theta power. Any model including Local Redundancy or only individual predictors performed worse (AIC $\geq 2383$ ). 


\section{Interim Discussion II}

The findings of our post-hoc computational modeling analysis replicate previous findings of increasing frontal theta power over time (Braithwaite et al., 2020) and show that surprise more so than complexity induced by the variability in the action demonstrations is linked to infants' higher theta power. The role of surprise in IDAs and infants' attentional processing is consistent with Event Segmentation Theory (Kurby \& Zacks, 2008; Zacks et al., 2007), which explains how we extract meaningful units from action streams. This theory states that, as observers, we use event models to constantly predict what happens next. When a stimulus is perceived as surprising, it leads to updating of the model in order to improve future predictability, also a core idea of the predictive-processing framework for infant learning (Köster et al., 2020). The temporary increase in processing at surprising moments thereby leads to more robust encoding and memory formation. While Event Segmentation Theory is focused on predictability troughs inherently present at action boundaries, parents artificially introduce predictability troughs in IDAs when interacting with their infants. This may suggest a similar underlying principle for both highlighting action goals in IDAs and segmenting events from a continuous action sequence (Baldwin et al., 2001). In other words, variable and thereby less predictable movements increase infants' attentional processing such that their attention level is high at the moment the goal of the action is demonstrated.

Additionally, the current findings are consistent with evidence showing that task-unrelated, unexpected stimuli increase children's arousal, which, in turn, leads to an improvement of task performance (Pozuelos et al., 2014). Increased movement variability in IDAs can also be interpreted as unrelated (or at most only marginally related) to the achievement of a goal, such as stacking rings on a peg. This additional layer of information tangential to the actual goal may increase the level of perceptual surprise, thereby increasing infants' arousal. Heightened attention might in turn lead to more efficient learning. Interestingly, the beneficial effect of task-unrelated stimuli for task performance disappears in 
Surprise induced by variability in IDA enhances infants' attention

late childhood (Pozuelos et al., 2014) and even reverses in adulthood, as the presence of task-unrelated stimuli hinders adults' performance on a given task (Wetzel, Widman \& Schröger, 2012). Hence, surprising stimuli might aid infants' learning in a unique way. 


\section{General Discussion}

Infants' attention is drawn to actions that are demonstrated in an infant-directed way to them (Brand \& Shallcross, 2008). Such IDAs also seem to benefit infants' action learning (Williamson \& Brand, 2014). The overarching question of the current research was how IDAs capture infants' attention and how they affect infants' subsequent exploration and action learning. In particular, we investigated the role variability plays in infants' attention to IDAs in an EEG study with 15-month-olds. As a neural index of attentional processing, we examined modulations in infants' frontal theta oscillations during IDAs and their link to subsequent exploration and learning. Consistent with our hypothesis, we show that variability in movements during IDAs rather than large movements per se attracts infants' attention. Furthermore, infants' frontal theta power during observation of the variable IDAs was related to their subsequent exploration and performance success of the demonstrated actions. More specifically, infants with higher frontal theta power were more likely to explore objects which were novel in the context of the current goal. For instance, when they had observed balls being moved into a bucket in the variable condition, they would first explore cups and rings when presented with a bucket. Furthermore, infants with higher frontal theta power were more likely to perform the target action correctly at least once. This link between frontal theta power of variable movements during IDAs and infants' learning is consistent with previous research investigating modulations in frontal theta power (Begus \& Bonawitz, 2020). Together, these results provide evidence suggesting an important role of variability in drawing infants' attention to and thereby foster their learning from IDAs.

To examine what is special about the information conveyed in the variable condition, we quantified the levels of surprise and complexity of each goal-directed movement in a post-hoc computational modelling analysis. The results not only replicate previous findings showing that frontal 
Surprise induced by variability in IDA enhances infants' attention

theta power increases with time (Braithwaite et al., 2020) but also demonstrate the key role of variabilityinduced surprise in increasing infant attention via theta power modulation.

\section{Neural processes: Open questions and future directions}

Based on our modeling findings, we might assume that surprise in movement variability elicits frontal theta power synchronization. This synchronization, in turn, leads to higher power during the subsequent goal attainment. The question arises whether the attentional benefit gained from the induced surprise has a temporal limitation and whether this limitation is based on the rhythm of theta and the size of the surprise. For example, would the time-window during which theta power is increased last longer if the surprise value was higher? Previous findings with adults suggest that the surprise systematically affects the magnitude of the theta increase (see e.g., Mas-Herrero \& Marco-Pallarés, 2014). Also, one might speculate that the frequency range of the oscillations determines the duration needed to return to a baseline level activity. These constraints in turn will have implications for teaching, both in terms of the degree of variability used and the temporal vicinity between the use of variability and the relevant aspect of the action. Future investigations are needed to address this question by systematically varying surprise magnitude and temporal arrangement between the (goal-unrelated) variability and the following (goalrelated) action unit.

Besides this, little is known about potential maturational effects of the medial prefrontal cortex (mPFC), which is the proposed neural generator of frontal theta oscillations (Ishii et al., 1999). Indeed, mPFC significantly develops throughout early childhood (Casey, Giedd \& Thomas, 2000). For instance, does structural maturation of this brain area underlie changes in processing of surprise and the beneficial effects of variability on task performance? Interestingly, despite the maturational changes of mPFC in the first years of life, research suggests that this area is already crucially involved in social-cognitive abilities from early on in infancy (Grossmann, 2013). Thus, it remains up to future research to unravel whether 
Surprise induced by variability in IDA enhances infants' attention

this might suggest any functional changes in processing surprise across development. Interdisciplinary research combining strengths of cognitive neuroscience techniques like fMRI and EEG as well as longitudinal developmental research is needed to address these questions.

\section{Implications for teaching novel actions}

Assuming that surprise is guiding infants' attention and driving their learning during IDAs several implications arise. By introducing variability (and thus surprise) in the movement that precedes the goal of the action, parents can utilize the attentional and mnemonic effects of surprise to teach their infant new actions more effectively. This might also explain the prominent use of repetitions in IDAs. Repetitions are omnipresent in parental IDAs (Brand, Baldwin \& Ashburn, 2002; van Schaik et al., 2020). They may serve two purposes at once, showing the consistency of the goal of the action while allowing for variability across repetitions in the goal-unrelated movement of the IDAs.

Another important consideration regards individual differences in surprise levels, since the level of surprise is based on the prior probability distribution. In other words, what is surprising to an infant may largely depend on the infant's prior experience. For instance, when an infant observes the same actions frequently, like lifting a cup in the same way over and over again, any deviation from that action will elicit surprise. In contrast, an infant who has hardly ever seen a particular movement or has had experience with variable movements, will react to the same action with a different level of surprise. When trying to optimize teaching of a new action to an infant it might therefore be crucial to individually adapt the degree of variability to induce surprise and thus increase attention appropriately. Parents, who typically know a large extent of their infants' prior experiences, have a good prerequisite to estimate their infant's surprise level to a particular situation. For people less familiar with an infant the information on infants' prior experience is not necessarily available. Still, a first behavioral indication of surprise (like changes in pupil size; Lin et al., 2018; Preuschoff, Hart, \& Einhauser, 2011) might help tune and adjust the 
IDAs to the individual infant. Another indicator allowing to dynamically adjust to the individual infant is their behavioral performance as an index of learning. In fact, Fukuyama and colleagues (2015) showed that parents spontaneously introduce more variability in their movement profile when their infant's behavioral performance is unrelated to the demonstrated action. This suggests that caregivers implement the use of variability as an attentional tool. While surprise induced by variability in IDAs certainly is not an exclusive means to draw infants' attention to an action, it seems both effective and practical in natural dyadic interactions with infants.

For demonstrating actions in infant-parent interactions, the emphasis is on making certain parts of one's actions variable. However, it is noteworthy that in a different context, dyadic interactions with infants benefit from the opposite, namely making oneself predictable. That is, success in joint action coordination is achieved by making one's actions temporally predictable (Vesper et al., 2011). Research with adults suggests that reducing one's movement variability is used as strategy for successful coordination with another person. Hence, when trying to coordinate with an infant, predictability in action performance is likely a more promising approach than introducing more variability. 


\section{Conclusions}

Together, our findings show that the use of variability in movements induces surprise which in turn serves as attentional tool during IDAs and which may have beneficial downstream effects for infants' action learning. As such, these findings advance our understanding of how IDAs capture infants' attention and how they affect infants' subsequent exploration and action learning. Making use of variability to elicit surprise might thus be a promising teaching tool to increase attention and foster memory formation. 


\section{Data Availability}

The EEG data processing scripts are publicly available on GitHub via https://github.com/marlenemeyer/EEG_pipeline_variability_in_infant_directed_actions. The data will only be publicly available after manuscript acceptance, in which case the persistent identifier that is currently reserved for this collection (https://doi.org/10.34973/0egj-ky51) will become effective.

\section{Funding Statement}

This research was supported by funding from the Dutch Research Council NWO to S.H. ("Loving to learn How curiosity drives cognitive development in young children"; VI.C.191.022).

\section{Conflict of Interest Disclosure}

The authors declare no competing interests.

\section{Ethics Approval Statement}

The current study adheres to the Declaration of Helsinki and was approved by the local ethics board.

\section{Acknowledgments}

We thank the families who participated in our study. We also would like to thank Didi Lamers and Robert Oostenveld for their help and support with converting the current dataset into BIDS format. 


\section{References}

Addyman, C., \& Mareschal, D. (2013). Local redundancy governs infants' spontaneous orienting to visualtemporal sequences. Child Development, 84(4), 1137-1144.

Baldwin, D. A., Baird, J. A., Saylor, M. M., \& Clark, M. A. (2001). Infants parse dynamic action. Child development, 72(3), 708-717.

Begus, K., \& Bonawitz, E. (2020). The rhythm of learning: theta oscillations as an index of active learning in infancy. Developmental Cognitive Neuroscience, 100810.

Begus, K., Southgate, V., \& Gliga, T. (2015). Neural mechanisms of infant learning: differences in frontal theta activity during object exploration modulate subsequent object recognition. Biology letters, 11(5), 20150041.

Brand, R. J., Baldwin, D. A., \& Ashburn, L. A. (2002). Evidence for 'motionese': modifications in mothers' infant-directed action. Developmental Science, 5(1), 72-83. doi 10.1111/1467-7687.00211

Brand, R. J., \& Shallcross, W. L. (2008). Infants prefer motionese to adult-directed action. Developmental Science, 11(6), 853-861. doi 10.1111/j.1467-7687.2008.00734.x

Brand, R. J., Shallcross, W. L., Sabatos, M. G., \& Massie, K. P. (2007). Fine-grained analysis of motionese: Eye gaze, object exchanges, and action units in infant-versus adult-directed action. Infancy, 11(2), 203-214.

BrainVision Recorder [Software]. (2020). Gliching, Germany: Brain Products GmbH.

Braithwaite, E. K., Jones, E. J., Johnson, M., \& Holmboe, K. (2020). Dynamic modulation of frontal theta power predicts cognitive ability in infancy. Developmental Cognitive Neuroscience, 100818. 
Surprise induced by variability in IDA enhances infants' attention

Casey, B. J., Giedd, J. N., \& Thomas, K. M. (2000). Structural and functional brain development and its relation to cognitive development. Biological Psychology, 54(1-3), 241-257.

Cavanagh, J. F., \& Frank, M. J. (2014). Frontal theta as a mechanism for cognitive control. Trends in Cognitive Sciences, 18(8), 414-421.

Clayton, M. S., Yeung, N., \& Kadosh, R. C. (2015). The roles of cortical oscillations in sustained attention. Trends in cognitive sciences, 19(4), 188-195.

Cullen, A. C., \& Frey, H. C. (1999). Probabilistic techniques in exposure assessment: a handbook for dealing with variability and uncertainty in models and inputs. Springer Science \& Business Media.

ELAN. (2018). [Computer software]. Nijmegen, the Netherlands: Max Planck Institute for Psycholinguistics. Retrieved from https://tla.mpi.nl/tools/tla-tools/elan/

Fukuyama, H., Qin, S., Kanakogi, Y., Nagai, Y., Asada, M., \& Myowa-Yamakoshi, M. (2015). Infant's action skill dynamically modulates parental action demonstration in the dyadic interaction. Developmental Science, 18(6), 1006-1013.

Ishii, R., Shinosaki, K., Ukai, S., Inouye, T., Ishihara, T., Yoshimine, T., ... \& Takeda, M. (1999). Medial prefrontal cortex generates frontal midline theta rhythm. Neuroreport, 10(4), 675-679.

Grossmann, T. (2013). The role of medial prefrontal cortex in early social cognition. Frontiers in Human Neuroscience, 7,340 .

Jamieson, R. K., \& Mewhort, D. J. K. (2005). The influence of grammatical, local, and organizational redundancy on implicit learning: an analysis using information theory. Journal of Experimental Psychology: Learning, Memory, and Cognition, 31(1), 9. 
Surprise induced by variability in IDA enhances infants' attention

Jensen, O., \& Tesche, C. D. (2002). Frontal theta activity in humans increases with memory load in a working memory task. European journal of Neuroscience, 15(8), 1395-1399.

Köster, M., Kayhan, E., Langeloh, M., \& Hoehl, S. (2020). Making sense of the world: Infant learning from a predictive processing perspective. Perspectives on psychological science, 1745691619895071.

Koterba, E. A., \& Iverson, J. M. (2009). Investigating motionese: The effect of infant-directed action on infants' attention and object exploration. Infant Behavior and Development, 32(4), 437-444.

Kurby, C. A., \& Zacks, J. M. (2008). Segmentation in the perception and memory of events. Trends in Cognitive Sciences, 12(2), 72-79.

Lin, H., Saunders, B., Hutcherson, C. A., \& Inzlicht, M. (2018). Midfrontal theta and pupil dilation parametrically track subjective conflict (but also surprise) during intertemporal choice. Neurolmage, 172, 838-852.

Mas-Herrero, E., \& Marco-Pallarés, J. (2014). Frontal theta oscillatory activity is a common mechanism for the computation of unexpected outcomes and learning rate. Journal of cognitive neuroscience, 26(3), 447-458.

Meyer, M., Endedijk, H. M., Van Ede, F., \& Hunnius, S. (2019). Theta oscillations in 4-year-olds are sensitive to task engagement and task demands. Scientific reports, 9(1), 1-11.

ManyBabies Consortium. (2020). Quantifying sources of variability in infancy research using the infantdirected-speech preference. Advances in Methods and Practices in Psychological Science, 3(1), 2452. 
Surprise induced by variability in IDA enhances infants' attention

Oudeyer, P. Y., Gottlieb, J., \& Lopes, M. (2016). Intrinsic motivation, curiosity, and learning: Theory and applications in educational technologies. In Progress in brain research (Vol. 229, pp. 257-284). Elsevier.

Orekhova, E. V., Stroganova, T. A., Posikera, I. N., \& Elam, M. (2006). EEG theta rhythm in infants and preschool children. Clinical neurophysiology, 117(5), 1047-1062.

Poli, F., Serino, G., Mars, R. B., \& Hunnius, S. (2020). Infants tailor their attention to maximize learning. Science advances, 6(39), eabb5053.

Pozuelos, J. P., Paz-Alonso, P. M., Castillo, A., Fuentes, L. J., \& Rueda, M. R. (2014). Development of attention networks and their interactions in childhood. Developmental Psychology, 50(10), 2405.

Preuschoff, K., t Hart, B. M., \& Einhauser, W. (2011). Pupil dilation signals surprise: Evidence for noradrenaline's role in decision making. Frontiers in neuroscience, 5, 115.

Räsänen, O., Kakouros, S., \& Soderstrom, M. (2018). Is infant-directed speech interesting because it is surprising? Linking properties of IDS to statistical learning and attention at the prosodic level. Cognition, 178, 193-206.

Schreiner, M. S., van Schaik, J. E., Sučević, J., Hunnius, S., \& Meyer, M. (2020). Let's talk action: Infantdirected speech facilitates infants' action learning. Developmental Psychology, 56(9), 1623.

Stasinopoulos, D. M., \& Rigby, R. A. (2007). Generalized additive models for location scale and shape (GAMLSS) in R. Journal of Statistical Software, 23(7), 1-46.

Tummeltshammer, K. S., \& Kirkham, N. Z. (2013). Learning to look: Probabilistic variation and noise guide infants' eye movements. Developmental Science, 16(5), 760-771. 
Surprise induced by variability in IDA enhances infants' attention

van Schaik, J. E., Meyer, M., van Ham, C. R., \& Hunnius, S. (2020). Motion tracking of parents' infant-versus adult-directed actions reveals general and action-specific modulations. Developmental Science, 23(1), e12869.

Vesper, C., van der Wel, R. P., Knoblich, G., \& Sebanz, N. (2011). Making oneself predictable: Reduced temporal variability facilitates joint action coordination. Experimental brain research, 211(3-4), 517-530.

Wetzel, N., Widmann, A., \& Schröger, E. (2012). Distraction and facilitation-two faces of the same coin?. Journal of Experimental Psychology: Human Perception and Performance, 38(3), 664.

Williamson, R. A., \& Brand, R. J. (2014). Child-directed action promotes 2-year-olds' imitation. Journal of Experimental Child Psychology, 118, 119-126.

Zacks, J. M., Speer, N. K., Swallow, K. M., Braver, T. S., \& Reynolds, J. R. (2007). Event perception: a mindbrain perspective. Psychological bulletin, 133(2), 273.

Zhang, Y., Koerner, T., Miller, S., Grice-Patil, Z., Svec, A., Akbari, D., ... \& Carney, E. (2011). Neural coding of formant-exaggerated speech in the infant brain. Developmental Science, 14(3), 566-581. 


\section{Figures}
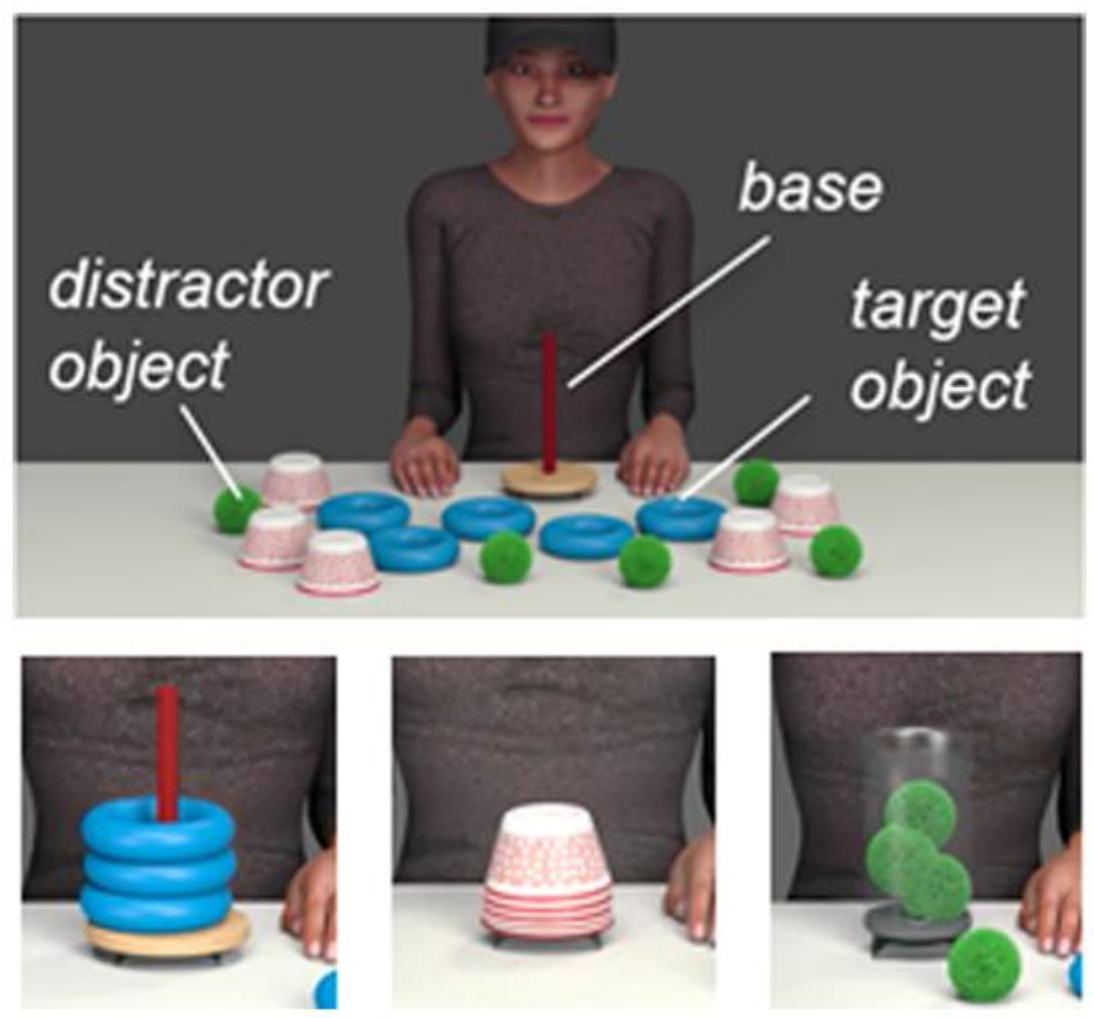

Figure 1. Top: Example of the video stimuli showing an avatar with a goal base (here peg) surrounded by corresponding target objects (here rings) and distractor objects (here balls and cups). The avatar acted on the goal base using the target objects. Bottom: Three different goal states representing the actions, i.e. stacking rings on a peg, building a tower with cups and putting balls in a bucket, were each shown in one condition (normal, high, variable), counterbalanced across participants. 

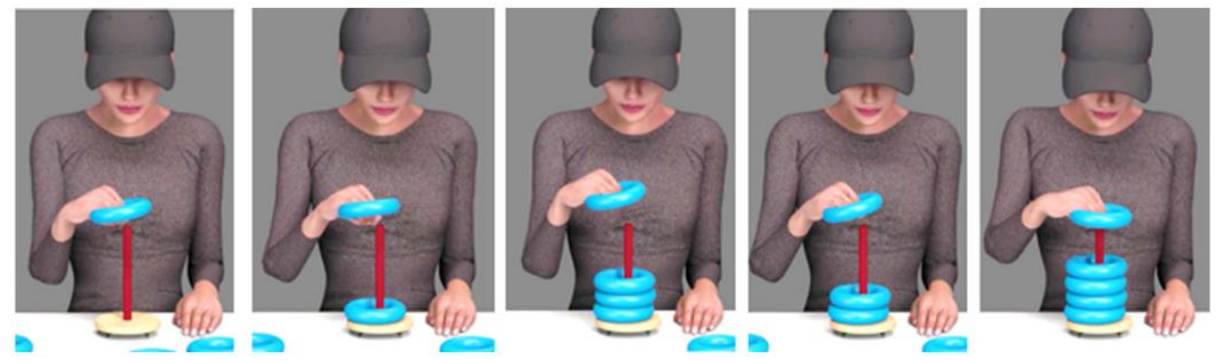

\section{normal}
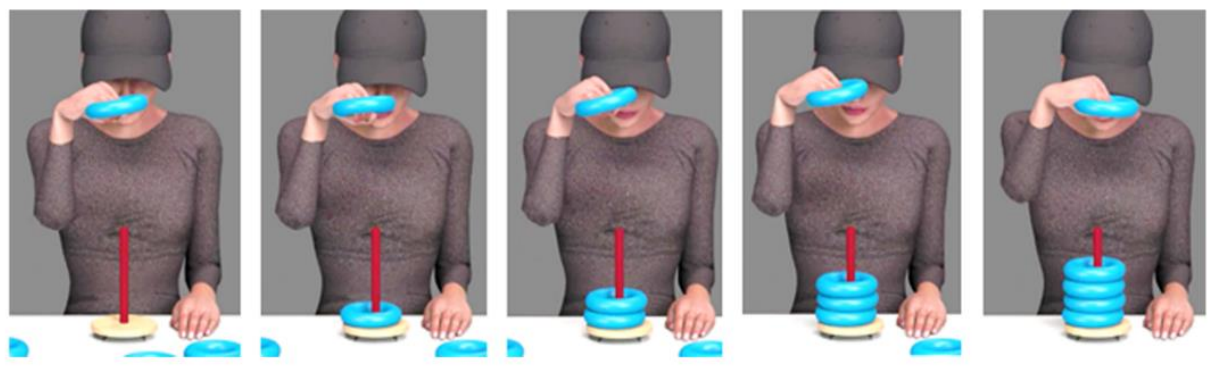

high
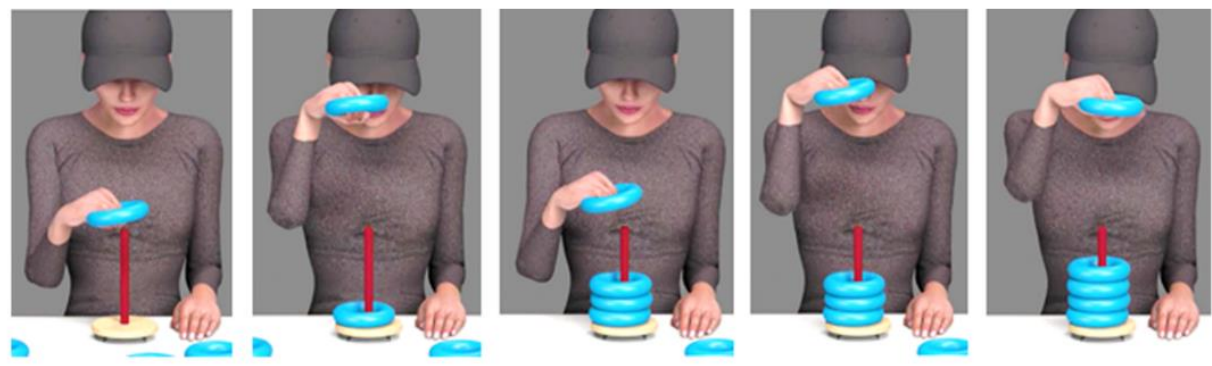

\section{variable}

Figure 2. Illustration of the three conditions of interest. In the normal amplitude condition (top) the action was demonstrated with normal amplitude movements for five subsequent times. In the high amplitude condition (middle) the action was demonstrated with high amplitude movements for five subsequent times. In the variable amplitude condition (bottom) the order of high and normal amplitude movements was varied during action demonstration. 


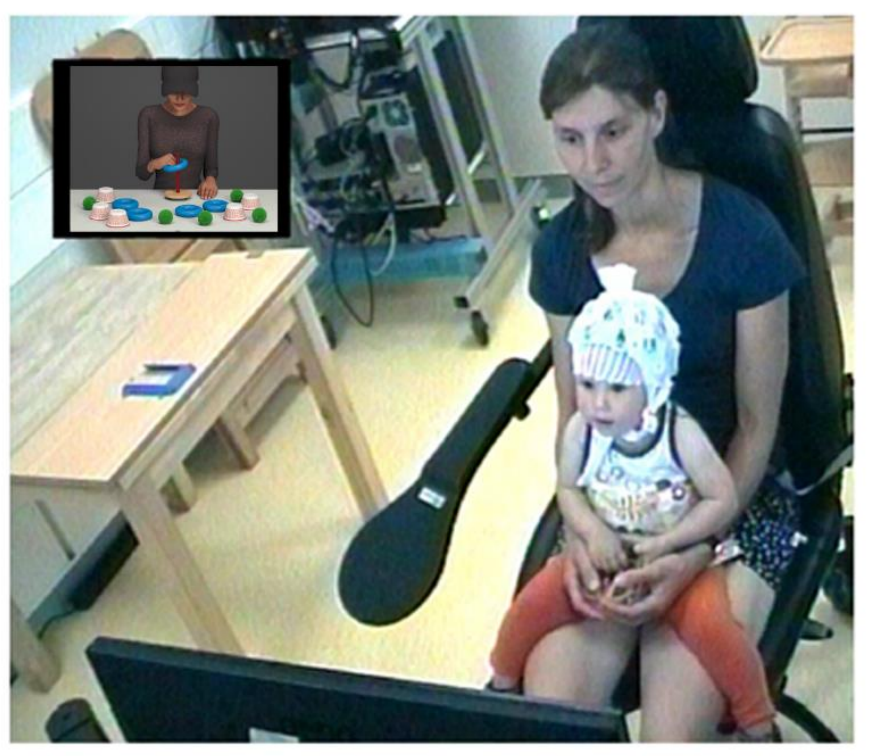

Demonstration

\section{All Objects}

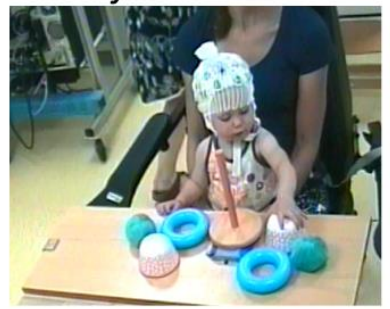

Target Objects Only

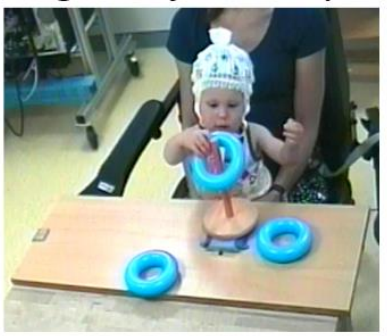

Exploration

Figure 3. Illustration of the experimental set-up. Left: Parent and infant participant during the demonstration phase in which novel actions are displayed using videos (see computer screen inlay in the top left corner). Infants' neural activity was measured during this phase using a 32 active channel EEG system. Right: Parent and infant participant during the exploration phase for which infants' behavior was video-coded offline. Top right: During the exploration phase, first each goal base (here peg) was presented with all objects (balls, rings, cups). Bottom right: After the availability of all objects, each goal base was presented with only the corresponding target objects (here rings). 
Surprise induced by variability in IDA enhances infants' attention
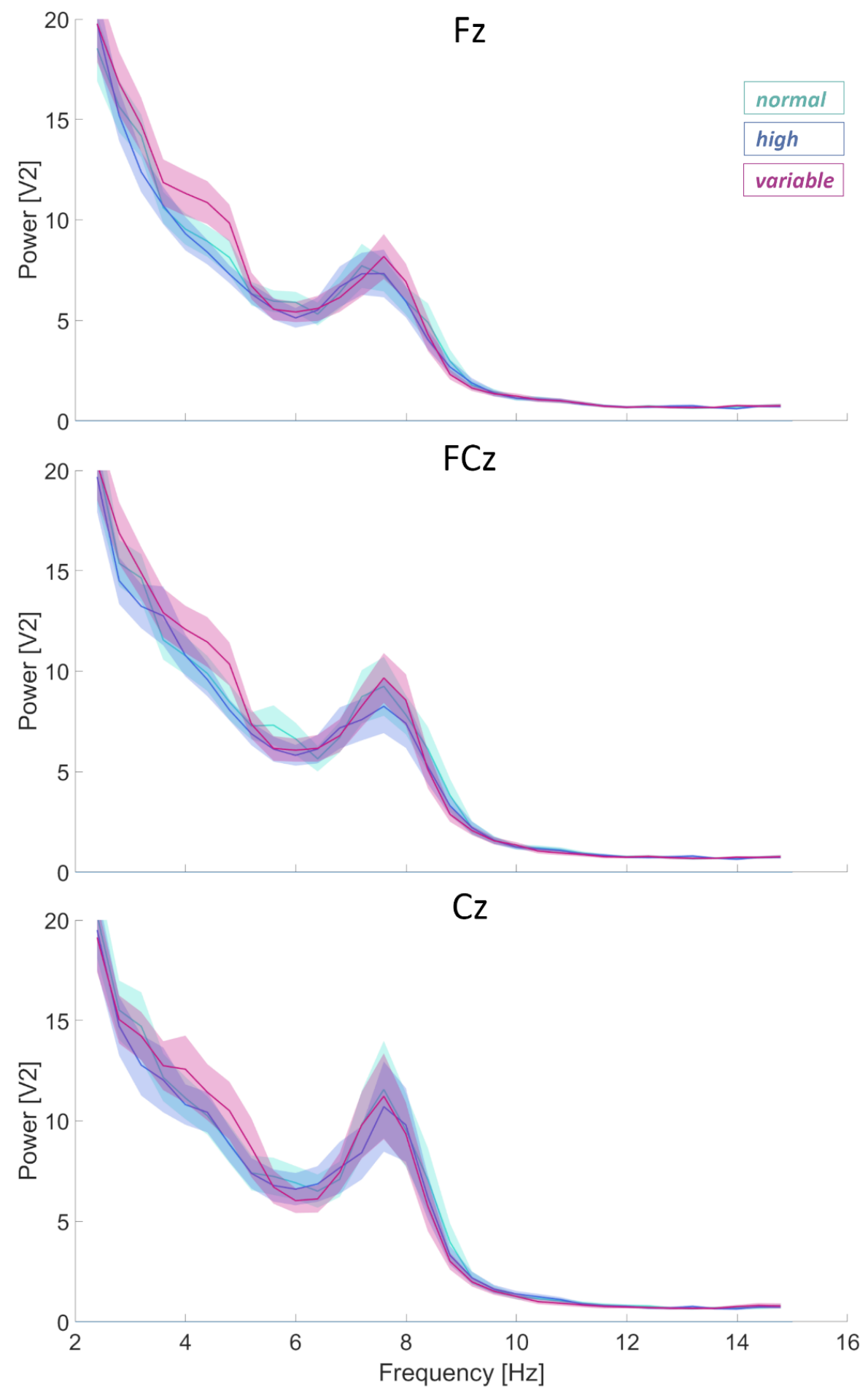

Figure 4. Power as a function of frequency is plotted per condition (normal, high, variable) for electrodes $F z, F C z$, and $C z$. Mean power values (solid lines) \pm 1 SE are displayed in accordingly shaded areas for all electrodes. 
Surprise induced by variability in IDA enhances infants' attention
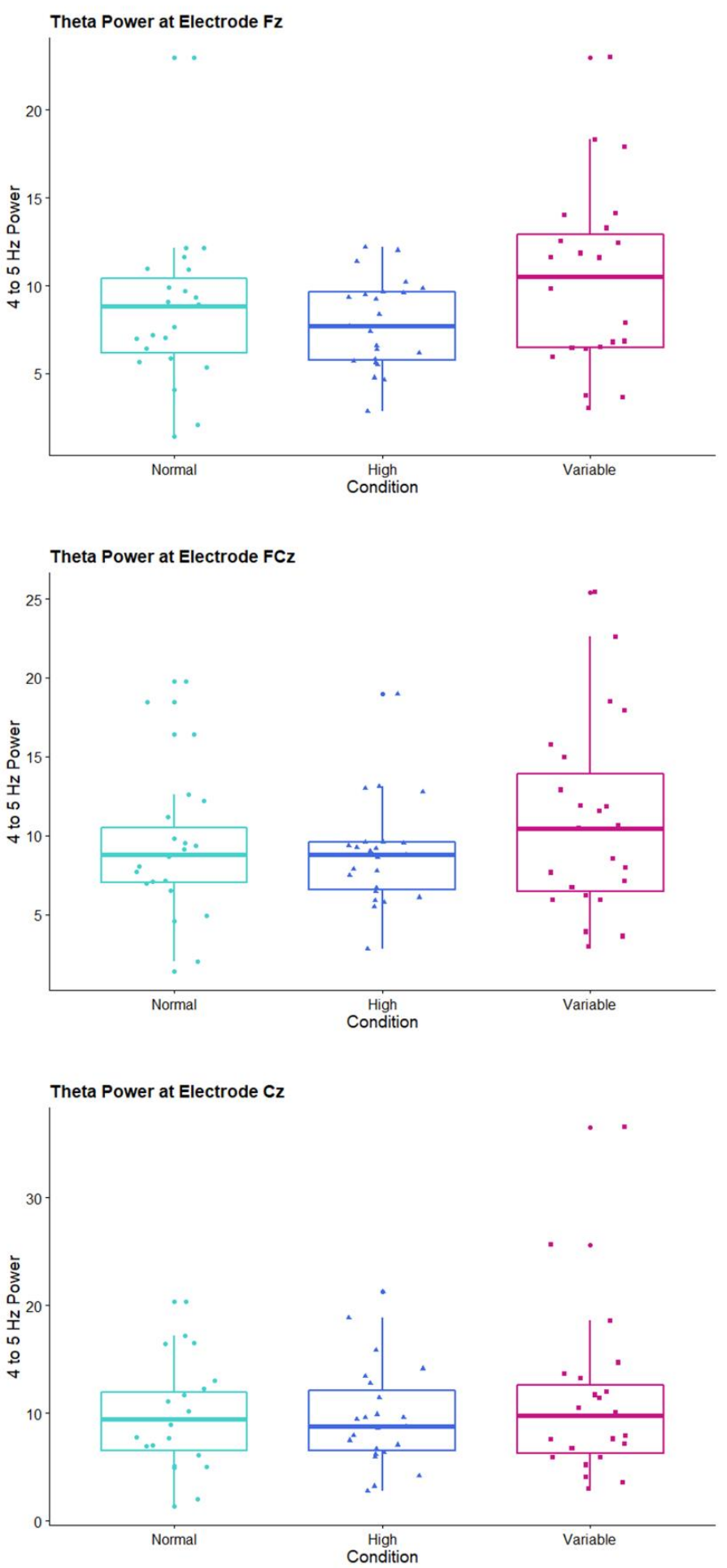

Figure 5. Results represented in boxplots overlayed with individuals' values. theta power values (4-5Hz) are displayed as a function of condition (normal, high, variable) for electrodes Fz (top), FCz (middle) and $C z$ (bottom). 
Surprise induced by variability in IDA enhances infants' attention

variable vs. normal

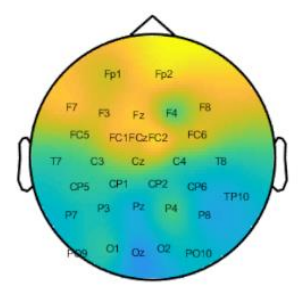

variable vs. high

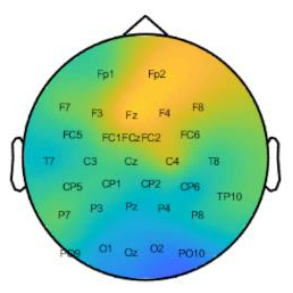

high vs. normal

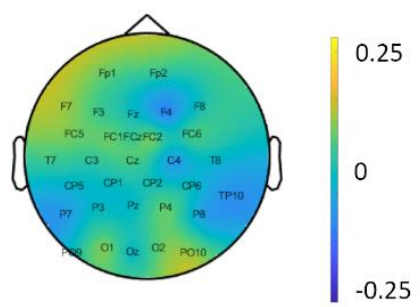

Figure 6. Topographic maps showing the difference in power of the theta frequency range (4-5Hz) between variable versus normal (left), variable versus high (middle), and high versus normal (right). Warm colors represent more power in theta for the variable (left and middle) and high (right) conditions respectively. 


\begin{tabular}{|c|c|c|c|c|}
\hline \multirow[b]{2}{*}{ Exploration Part } & \multirow[b]{2}{*}{ Variable } & \multicolumn{3}{|c|}{ Model } \\
\hline & & $b$ & $S E b$ & $\beta$ \\
\hline \multirow[t]{2}{*}{ All Objects } & Object First Touched & -4.83 & 2.0 & $-.49^{*}$ \\
\hline & Successful Target Performance & -4.01 & 2.47 & -.37 \\
\hline \multirow[t]{3}{*}{$\begin{array}{l}\text { Target Objects } \\
\text { Only }\end{array}$} & Successful Target Performance & 5.54 & 2.27 & $.57 *$ \\
\hline & $R^{2}$ & & 0.36 & \\
\hline & $F$ for change in $R^{2}$ & & $3.49^{*}$ & \\
\hline
\end{tabular}

Table 1. Linear regression model of frontal theta power during the variable condition and infants' exploration and learning behavior for presentation of the goal base associated with this condition. 
Surprise induced by variability in IDA enhances infants' attention
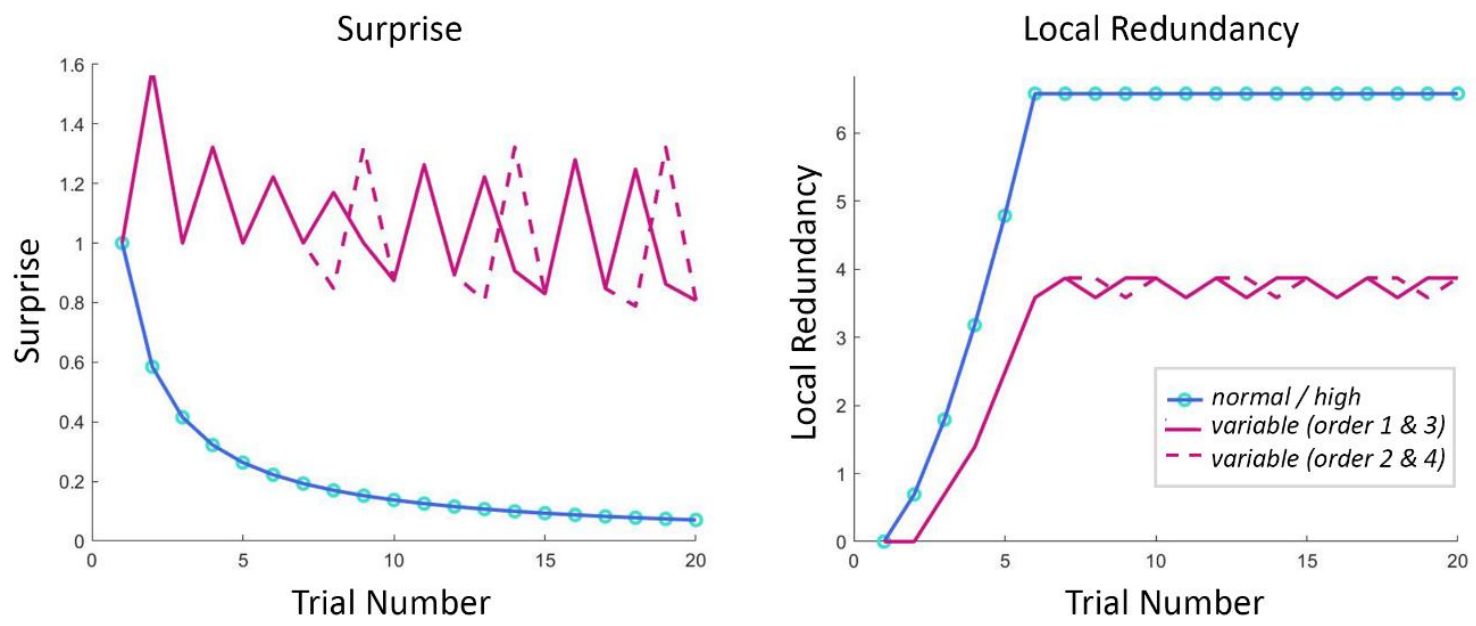

Figure 7. Surprise (left) and Local Redundancy (right) as a function of trial number in example sequences of the normal, high, and variable condition. The variable condition had several counterbalancing orders. The values were computed considering the conditions as independent of each other. Trials of each condition were treated as consecutive events in a sequence of goal-directed movements making the assumption that infants dissociate the three different actions despite their presentation in separate blocks of videos. 
Surprise induced by variability in IDA enhances infants' attention

\section{Table 2: Model comparison}

\begin{tabular}{lll}
\hline Model & AIC & $\Delta$ AIC \\
\hline I, T & 2380 & 0 \\
LR, T & 2383 & 3 \\
T & 2386 & 6 \\
I & 2391 & 11 \\
LR & 2394 & 14 \\
\hline
\end{tabular}

Table 2. Model comparison including the following variables $I=$ Surprise, $L R=$ Local Redundancy, $T=$ Trial Number. Lower AIC values indicate better model fit. $\triangle A I C$ is computed as the absolute difference between the best model and any other model. 